\title{
Kidney failure, CKD progression and mortality after nephrectomy
}

\author{
Robert J. Ellis ${ }^{1,2,3}$ - Anne Cameron ${ }^{2,4} \cdot$ Glenda C. Gobe ${ }^{2,3,4} \cdot$ Vishal Diwan $^{2,4} \cdot$ Helen G. Healy ${ }^{4,5,6} \cdot$ Jeremy Lee ${ }^{1}$. \\ Ken-Soon $\operatorname{Tan}^{2,4,7}$. Sree Venuthurupalli ${ }^{2,4,8}$. Jianzhen Zhang ${ }^{2,4}$. Wendy E. Hoy ${ }^{2,4}$ on behalf of the NHMRC CKD.CRE, \\ the CKD.QLD Collaborative
}

Received: 31 August 2021 / Accepted: 11 January 2022 / Published online: 27 January 2022

(c) The Author(s) 2022

\begin{abstract}
Purpose This study tested the hypothesis that progression of chronic kidney disease (CKD) is less aggressive in patients whose primary cause of CKD was nephrectomy, compared with non-surgical causes.

Methods A sample of 5983 patients from five specialist nephrology practices was ascertained from the Queensland CKD Registry. Rates of kidney failure/death were compared on primary aetiology of CKD using multivariable Cox proportional hazards models. CKD progression was compared using multivariable linear and logistic regression analyses.

Results Of 235 patients with an acquired single kidney as their primary cause of CKD, 24 (10\%) and 38 (17\%) developed kidney failure or died at median [IQR] follow-up times of 12.9 [2.5-31.0] and 33.6 [18.0-57.9] months after recruitment. Among patients with an eGFR $<45 \mathrm{~mL} / \mathrm{min}$ per $1.73 \mathrm{~m}^{2}$ at recruitment, patients with diabetic nephropathy and PCKD had the highest rates (per 1000 person-years) of kidney failure (107.8, 95\% CI 71.0-163.8; 75.5, 95\% CI 65.6-87.1); whereas, patients with glomerulonephritis and an acquired single kidney had lower rates (52.9, 95\% CI 38.8-72.1; 34.6, 95\% CI 20.5-58.4, respectively). Among patients with an eGFR $\geq 45 \mathrm{~mL} / \mathrm{min}$ per $1.73 \mathrm{~m}^{2}$, those with diabetic nephropathy had the highest rates of kidney failure (16.6, 95\% CI 92.5-117.3); whereas, those with glomerulonephritis, PCKD and acquired single kidney had a lower risk (11.3, 95\% CI 7.1-17.9; 11.7, 95\% CI 3.8-36.2; 10.7, 95\% CI 4.0-28.4, respectively).

Conclusion Patients who developed CKD after nephrectomy had similar rates of adverse events to most other causes of CKD, except for diabetic nephropathy which was consistently associated with worse outcomes. While CKD after nephrectomy is not the most aggressive cause of kidney disease, it is by no means benign, and is associated with a tangible risk of kidney failure and death, which is comparable to other major causes of CKD.
\end{abstract}

Keywords Chronic kidney disease $\cdot$ Nephrectomy $\cdot$ Kidney failure

Robert J. Ellis

robert.ellis2@health.qld.gov.au

Princess Alexandra Hospital, Brisbane, QLD, Australia

2 Faculty of Medicine, University of Queensland, Brisbane, QLD, Australia

3 Kidney Disease Research Collaborative, Translational Research Institute, 37 Kent Street, Woolloongabba, Brisbane, QLD 4102, Australia

4 NHMRC CKD.CRE and the CKD.QLD Collaborative, University of Queensland, Brisbane, QLD, Australia

5 Kidney Health Service, Royal Brisbane and Women's Hospital, Brisbane, QLD, Australia

6 Conjoint Internal Medicine Laboratory, Pathology Queensland, Brisbane, QLD, Australia

7 Department of Nephrology, Logan Hospital, Logan, QLD, Australia

8 Renal Service, Ipswich Hospital, Brisbane, QLD, Australia

\section{Introduction}

Kidney reduction surgery, removal of all (radical/total nephrectomy) or part (partial nephrectomy) of the kidney, is the most common management approach for suspicious kidney masses, and curative for most localised kidney cancers [1]. While providing good oncological control, the surgical reduction of functional kidney parenchyma can contribute to reduced kidney function, characterised by declining estimated glomerular filtration rate (eGFR). Many patients with previously normal kidney function pre-nephrectomy will be classed as having chronic kidney disease (CKD) postoperatively, based on eGFR criteria. In the only randomised controlled trial comparing radical with partial nephrectomy, $85.7 \%$ of patients managed with radical nephrectomy and $64.7 \%$ of patients managed with partial nephrectomy had 
an eGFR $<60 \mathrm{~mL} / \mathrm{min}$ per $1.73 \mathrm{~m}^{2}$ (stage $\geq 3 \mathrm{a}$ CKD) at a median follow-up time of 6.7 years postoperatively [2]. While tumours are the most frequent indication for nephrectomy, other common indications include trauma, infection, obstruction, and donation.

Several authors have reported that, compared with 'medical' causes of nephron loss, surgical nephron reduction is associated with lower rates of CKD progression, kidney failure, and death [3,4]. One interpretation is that medical kidney disease is associated with continuous exposure to the underlying pathophysiology causing nephron loss, whereas the surgical insult is time-limited and, presumably, not associated with chronic pathophysiological processes [5]. This interpretation is not grounded in evidence. The cohort of medical causes of CKD is extremely broad, with considerable heterogeneity in the cause of kidney disease, rates of CKD progression, and severity of kidney impairment [6]. Many patients with CKD will not experience obvious declines in kidney function over time or progress to kidney failure [7-9]. Notwithstanding, all patients with stages 3b, 4 and 5 CKD have higher risks of mortality compared with patients who have stages 1,2 and $3 \mathrm{a}$ [10], and this risk is inversely proportional to eGFR [11].

This study aimed to test the hypothesis that rates of kidney failure, mortality, and CKD progression are lower in patients whose CKD is due to surgical nephron reduction (acquired single kidney) compared with other medical causes, within a population of pre-dialysis patients known to specialty nephrology services in Queensland, Australia.

\section{Patients and methods}

\section{Study population}

Data were extracted for 5983 patients enrolled in the Queensland CKD Registry (CKD.QLD) from five clinical sites across Queensland, Australia: Royal Brisbane and Women's Hospital $(n=1643)$, Logan Hospital $(n=1534)$, Mackay Base Hospital $(n=680)$, Toowoomba Hospital $(n=1470)$, and Townsville University Hospital $(n=656)$ [12]. Patients were recruited between June 2011 and June 2018; data were extracted from the registry in January 2020. Inclusion criteria for the registry are a diagnosis of CKD and care under a specialist nephrology practice in Queensland.

Patients were excluded if they had an unclear, unknown, or unspecified primary kidney disease $(n=625)$, dates were ambiguous or missing $(n=106)$, baseline eGFR data were missing $(n=27)$, or if they had kidney failure (eGFR $<15 \mathrm{~mL} / \mathrm{min}$ per $\left.1.73 \mathrm{~m}^{2}\right)$ at the time of recruitment $(n=345)$. There were 4,880 patients in the final sample. Human research ethics approval was obtained from the Royal Brisbane and Women's Hospital (HREC/15/QRBW/294) and the University of Queensland (2011000029) and a Public Health Act Approval (QCOS/029817/RD006802) for Queensland Health data linkages. All participants provided written informed consent before being included in the CKD. QLD registry.

The registry is informed by an association with the Statistical Analysis and Linkage Unit (SALU), Queensland Health, who link the CKD.QLD registry patient cohort to the following collections under a Public Health Act approval: Queensland Hospital Admitted Patient Data Collection (QHAPDC), Kidney replacement therapy (KRT), Queensland Activity Based Funding (ABF) Model Output Data, Death Registrations from the (Qld) Registry of Births, Deaths and Marriages and Cause of Death Unit Record File from the Australian Coordinating Registry, informing on institution of KRT and deaths.

\section{Exposures}

Age at recruitment was calculated from the date of birth and date of recruitment. Date of recruitment was defined as the date informed consent was given for inclusion, capturing both incident and prevalent cases. Diabetes mellitus and hypertension were classified as yes, no, or missing. Smoker status was classified as never, former or current smoker, or missing. The primary kidney disease was grouped into five categories (diabetic nephropathy, glomerulonephritis, polycystic kidney diseases [PCKD], acquired single kidney, or other) based on coded diagnosis and free-text fields in the database, where the person's treating nephrologist assigned the primary cause(s) of CKD based on clinical assessment. The acquired single kidney group included those where nephrectomy was listed as the primary cause of CKD. An ancillary chart review of this group was undertaken to determine the date and indication for nephrectomy. Baseline eGFR was determined from serum creatinine taken at the time of recruitment; eGFR at 12 and 24 months after recruitment was recorded if available in the patient's electronic medical records. The CKD-EPI equation was used to calculate eGFR. Data collection has been described previously [12].

\section{Outcomes}

The primary outcome of interest was a composite of time to the development of kidney failure (defined as eGFR persistently $<15 \mathrm{~mL} / \mathrm{min}$ per $1.73 \mathrm{~m}^{2}$ ) or commencement of KRT. Secondary outcomes included time to all-cause mortality, change in eGFR, and CKD progression. The CKD.QLD registry provided event data for kidney failure, KRT, and death, after performing data linkages of state electronic data sets. Change in eGFR was calculated over at least a 12-month period, and defined as the difference between baseline eGFR 
at recruitment, and eGFR at either 12 or 24 months, depending on the most recent value available. Progressive CKD was defined as an annual decrease in eGFR of at least $5 \mathrm{~mL} / \mathrm{min}$ per $1.73 \mathrm{~m}^{2}$ without subsequent recovery, the development of kidney failure, or the requirement for KRT within two years of recruitment.

\section{Statistical analysis}

Baseline characteristics were presented descriptively. The association between primary kidney disease and time to kidney failure/KRT (primary outcome) and all-cause mortality (secondary outcome) was analysed using Cox proportional hazards models. When evaluating kidney failure/ KRT, participants were censored on either the site-specific date that the database was updated, last date the person was seen before being lost to follow-up, or date of death. When evaluating all-cause mortality, participants were censored on the date the database was updated or date lost to follow-up. All analyses were stratified by baseline eGFR $\left(<45\right.$ and $\geq 45 \mathrm{~mL} / \mathrm{min}$ per $\left.1.73 \mathrm{~m}^{2}\right)$, which was considered a clinically significant cut-point [13]. The reference group was defined as the cohort with diabetic nephropathy as primary cause of CKD and a baseline eGFR $\geq 45 \mathrm{~mL} / \mathrm{min}$ per $1.73 \mathrm{~m}^{2}$. A sensitivity analysis evaluated time to kidney failure/KRT with death as a competing event, using Fine and Gray's proportional sub-hazards model. Change in eGFR was analysed using multivariable linear regression, stratifying analysis by baseline eGFR. The reference group was defined as the cohort with an acquired single kidney. Unlike the other analyses, comparison was only made within instead of between eGFR strata, as the decreased accuracy of the CKD-EPI equation at lower values for serum creatinine may introduce bias if directly comparing the two strata using the same definition for eGFR change [14]. Progressive CKD was analysed using multivariable logistic regression, stratified by baseline eGFR. The reference group was defined as the cohort with diabetic nephropathy and an eGFR $<45 \mathrm{~mL} / \mathrm{min}$ per $1.73 \mathrm{~m}^{2}$. In all multivariable models, confounders were identified using directed acyclic graphs. A supplementary analysis of patients with an acquired single kidney for whom a date and indication for nephrectomy was able to be determined from chart review; indication and year were compared descriptively by whether or not the patient progressed to kidney failure. Analysis was performed using Stata 14.2 (StataCorp, College Station, TX).

\section{Results}

This study included 4,880 general nephrology patients from Queensland, Australia (Table 1). At the time when data were extracted, $633(12 \%)$ and 1,267 (24\%) participants
Table 1 Baseline characteristics of study population

\begin{tabular}{ll}
\hline Variable & $(n=4880)$ \\
\hline Age, years & \\
Median [IQR] & $68.2[56.4-76.5]$ \\
Sex $(n, \%)$ & \\
Female & $2304(47)$ \\
Male & $2576(53)$ \\
Diabetes mellitus $(n, \%)$ & \\
No & $2448(50)$ \\
Yes & $2368(49)$ \\
Missing & $64(1)$ \\
Hypertension $(n, \%)$ & \\
No & $811(17)$ \\
Yes & $3516(72)$ \\
Missing & $553(11)$ \\
Smoker status $(n, \%)$ & \\
Never & $876(18)$ \\
Former & $1155(24)$ \\
Current & $452(9)$ \\
Missing & $2397(49)$ \\
Baseline eGFR & \\
Median $[$ IQR] & \\
Range & $39[29-54]$ \\
Primary kidney disease $(n, \%)$ & $15-94$ \\
Diabetic nephropathy & \\
Glomerulonephritis & $1238(25)$ \\
PCKD & $616(13)$ \\
Acquired single Kidney & $139(3)$ \\
Other & $221(5)$ \\
\hline & $2666(55)$ \\
\hline
\end{tabular}

eGFR estimated glomerular filtration rate (in $\mathrm{mL} / \mathrm{min}$ per $1.72 \mathrm{~m}^{2}$ ), $I Q R$ interquartile range, $P C K D$ polycystic kidney diseases

had developed kidney failure/commenced KRT, or died at median [interquartile range (IQR)] follow-up times of 19.2 [7.8-33.6] and 31.6 [16.0-48.9] months following recruitment. The primary indication for nephrology referral was an acquired single kidney for 235 (5\%) participants; of these, 24 (10\%) and 38 (17\%) developed kidney failure/ required KRT or died at median [IQR] follow-up times of 12.9 [2.5-31.0] and 33.6 [18.0-57.9] months, respectively, following recruitment.

Rates of kidney failure/KRT were compared by primary aetiology of CKD and dichotomised to eGFR (at recruitment) $<45$ and $\geq 45 \mathrm{~mL} / \mathrm{min}$ per $1.73 \mathrm{~m}^{2}$ (Table 2; Fig. 1). Among participants with an eGFR $<45 \mathrm{~mL} / \mathrm{min}$ per $1.73 \mathrm{~m}^{2}$, those with PCKD had the highest rates (per 1,000 personyears) of kidney failure/KRT (107.8, 95\% confidence interval [CI] 71.0-163.8). The diabetic nephropathy cohort had the next highest $(75.5,95 \%$ CI $65.6-87.1)$, followed by the glomerulonephritis cohort $(52.9,95 \%$ CI $38.8-72.1)$. The acquired single kidney cohort had the lowest crude rate of 
Table 2 Kidney failure and death compared by primary kidney disease $(n=4,880)$

\begin{tabular}{|c|c|c|c|c|c|}
\hline & Person-years & $\begin{array}{l}\text { Number of } \\
\text { events }\end{array}$ & $\operatorname{Rate}^{\mathrm{a}}(95 \%$ CI $)$ & Crude HR $(95 \% \mathrm{CI})$ & Adjusted HR $(95 \% \mathrm{CI})$ \\
\hline \multicolumn{6}{|l|}{ Kidney failure ${ }^{\mathrm{b}}$} \\
\hline \multicolumn{6}{|l|}{ Baseline eGFR $<45$} \\
\hline Diabetic nephropathy & 2516 & 190 & $75.5(65.5-87.1)$ & $4.6(3.0-6.9)$ & $5.3(3.5-8.1)$ \\
\hline Glomerulonephritis & 757 & 40 & $52.9(38.8-72.1)$ & $3.2(1.9-5.2)$ & $2.8(1.6-4.9)$ \\
\hline PCKD & 204 & 22 & $107.8(71.0-163.8)$ & $6.5(3.7-11.4)$ & $6.3(3.4-11.6)$ \\
\hline Acquired single kidney & 405 & 14 & $34.6(20.5-58.4)$ & $2.1(1.1-4.0)$ & $3.3(1.7-6.5)$ \\
\hline Other & 5570 & 139 & $25.0(21.1-29.5)$ & $1.5(0.9-4.0)$ & $2.3(1.4-3.6)$ \\
\hline \multicolumn{6}{|l|}{ Baseline eGFR $\geq 45$} \\
\hline Diabetic nephropathy & 1564 & 26 & $16.6(11.3-24.4)$ & 1.0 & 1.0 \\
\hline Glomerulonephritis & 1595 & 18 & $11.3(7.1-17.9)$ & $0.7(0.4-1.2)$ & $0.4(0.2-0.8)$ \\
\hline PCKD & 257 & 3 & $11.7(3.8-36.2)$ & $0.6(0.2-1.8)$ & $0.4(0.1-1.3)$ \\
\hline Acquired single kidney & 375 & 4 & $10.7(4.0-28.4)$ & $0.6(0.2-1.8)$ & $0.7(0.2-1.9)$ \\
\hline Other & 3335 & 12 & $3.6(2.0-6.3)$ & $0.2(0.1-0.4)$ & $0.2(0.1-0.4)$ \\
\hline \multicolumn{6}{|l|}{ Death $^{\mathrm{c}}$} \\
\hline \multicolumn{6}{|l|}{ Baseline eGFR $<45$} \\
\hline Diabetic nephropathy & 2630 & 274 & $104.2(92.5-117.3)$ & $3.0(2.3-4.0)$ & $2.3(1.7-3.1)$ \\
\hline Glomerulonephritis & 782 & 41 & $52.4(38.6-71.2)$ & $1.4(1.0-2.2)$ & $2.0(1.3-3.0)$ \\
\hline PCKD & 206 & 5 & $24.2(10.1-58.2)$ & $0.7(0.3-1.8)$ & $1.0(0.4-2.5)$ \\
\hline Acquired single kidney & 424 & 30 & 70.7 (49.4-101.1) & $2.0(1.3-3.1)$ & $1.5(1.0-2.4)$ \\
\hline Other & 5.793 & 574 & 99.1 (91.3-107.6) & $2.8(2.2-3.7)$ & $2.1(1.6-2.8)$ \\
\hline \multicolumn{6}{|l|}{ Baseline eGFR $\geq 45$} \\
\hline Diabetic nephropathy & 1577 & 57 & $36.1(27.9-46.8)$ & 1.0 & 1.0 \\
\hline Glomerulonephritis & 1605 & 15 & $9.3(5.6-15.5)$ & $0.2(0.1-0.4)$ & $0.6(0.3-1.0)$ \\
\hline PCKD & 260 & 3 & $11.6(3.7-35.8)$ & $0.3(0.1-1.1)$ & $1.0(0.3-3.2)$ \\
\hline Acquired single kidney & 382 & 8 & $20.9(10.5-41.9)$ & $0.6(0.3-1.2)$ & $0.9(0.4-1.9)$ \\
\hline Other & 3377 & 102 & $30.2(24.9-36.8)$ & $0.8(0.6-1.1)$ & $1.0(0.8-1.5)$ \\
\hline
\end{tabular}

Hazard ratios (HR) and 95\% confidence intervals (CI) estimated using Cox proportional hazards models $e G F R$ estimated glomerular filtration rate (in $\mathrm{mL} / \mathrm{min}$ per $1.73 \mathrm{~m}^{2}$ ), $P C K D$ polycystic kidney diseases

${ }^{a}$ Unadjusted rate per 1000 person-years

${ }^{\mathrm{b}}$ Multivariable model adjusted for age, sex, diabetes mellitus, and hypertension

${ }^{\mathrm{c}}$ Multivariable model adjusted for age, sex, diabetes mellitus, hypertension, and smoker status

kidney failure (34.6, 95\% CI 20.5-58.4) among aetiological groups. These rankings were preserved when evaluating point estimates for the crude hazard ratios for risk of kidney failure/KRT; however, there was no difference between the acquired single kidney and glomerulonephritis groups when adjustment was made for age, sex, and the presence of diabetes/hypertension. Among the eGFR $\geq 45 \mathrm{~mL} / \mathrm{min}$ per $1.73 \mathrm{~m}^{2}$ groups, the highest rates of kidney failure/KRT was seen in the diabetic nephropathy cohort $(16.6,95 \%$ CI 92.5-117.3), with similar rates in the glomerulonephritis, PCKD, and acquired single kidney cohorts $(11.3,95 \%$ CI 7.1-17.9; 11.7, 95\% CI 3.8-36.2; 10.7, 95\% CI 4.0-28.4, respectively). Crude hazard ratios for kidney failure/KRT were similar across the aetiological groups. After adjusting for age, sex, and diabetes/hypertension, the point estimate for those with an acquired single kidney was higher than all groups except diabetic nephropathy. Similar patterns were observed in the analysis considering death as a competing event (Supplementary Table 1).

Rates of death in participants with an acquired single kidney were 70.7 (95\% CI 49.4-101.1) in the eGFR $<45 \mathrm{~mL} /$ min per $1.73 \mathrm{~m}^{2}$ group, and 20.9 (95\% CI 10.5-41.9) in the eGFR $\geq 45 \mathrm{~mL} / \mathrm{min}$ per $1.73 \mathrm{~m}^{2}$ group (Table 2). Differences in risk of death across the aetiological groups disappeared when assessing hazard ratios adjusted for age, sex, smoker status, and the presence of diabetes and hypertension.

There were similar relationships among the aetiological groups when evaluating CKD progression and change in eGFR as there was when evaluating kidney failure (Supplementary Table 2).

Participants in the acquired single kidney cohort who developed kidney failure/KRT tended to be male, have 


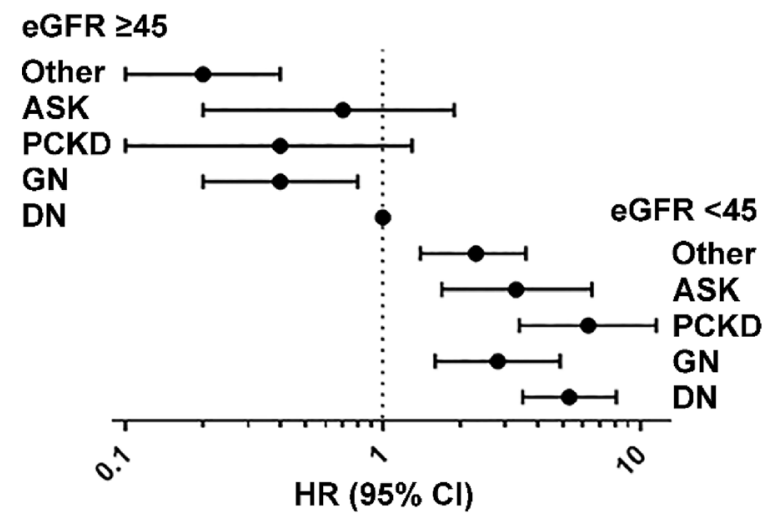

(Favours Kidney Failure)

Fig. 1 Likelihood of kidney failure compared by primary cause of CKD. Forest plot comparing the hazard ratio (HR) and 95\% confidence interval (CI) for the development of kidney failure after recruitment compared by primary aetiology of chronic kidney disease (CKD). Results stratified by estimated glomerular filtration rate (eGFR; in $\mathrm{mL} / \mathrm{min}$ per $1.73 \mathrm{~m}^{2}$ ). $A S K$ acquired single kidney; $D N$ diabetic nephropathy, $G N$ glomerulonephritis, $P C K D$ polycystic kidney disease

comorbidities, such as hypertension and diabetes mellitus, and a lower eGFR at recruitment, compared with those who did not develop kidney failure/KRT (Table 3). There was no substantial difference between indication or timing of nephrectomy and development of kidney failure (Supplementary Table 3 and 4).

\section{Discussion}

This study aimed to evaluate rates of kidney failure/KRT, mortality, and CKD progression in people with an acquired single kidney and managed in the clinical practices of specialist nephrologists. These were compared with other patients managed in these practices with CKD of other aetiologies.

As expected, likelihood of kidney failure/KRT was higher in the eGFR $<45 \mathrm{~mL} / \mathrm{min}$ per $1.72 \mathrm{~m}^{2}$ group compared with those with a higher baseline eGFR. Participants in the diabetic nephropathy cohort were particularly likely to reach this endpoint compared with those who had an acquired single kidney, regardless of baseline eGFR. This is not surprising, given the multiple pathophysiological mechanisms involved in diabetes mellitus, including recurrent and/or persistent hyperglycaemia, macro- and microvascular atherosclerotic changes, and cardiovascular disease, in addition to processes activated by nephron loss $[15,16]$.

Participants with glomerulonephritis had very similar risks of kidney failure/KRT compared with participants with an acquired single kidney. Notwithstanding, the estimates were relatively imprecise with overlapping confidence
Table 3 Baseline characteristics of patients with an acquired single kidney

\begin{tabular}{llll}
\hline Variable & \multicolumn{2}{c}{ Developed kidney failure } & \multirow{2}{*}{$p$ value } \\
\cline { 2 - 3 } & No $(n=203)$ & Yes $(n=18)$ & \\
\hline Age, years & & & \\
Median [IQR] & $67.2[59.4-76.6]$ & $67.1[59.8-72.7]$ & 0.84 \\
Sex $(n, \%)$ & & \\
Female & $93(46)$ & $4(22)$ & \\
Male & $110(54)$ & $14(78)$ & \\
Diabetes mellitus $(n, \%)$ & & \\
No & $125(62)$ & $9(50)$ & \\
Yes & $74(36)$ & $8(44)$ & 0.45 \\
Missing & $4(2)$ & $1(6)$ & \\
Hypertension $(n, \%)$ & & \\
No & $52(26)$ & $2(11)$ & \\
Yes & $119(59)$ & $17(89)$ & \\
Missing & $32(16)$ & - & \\
Smoker status $(n, \%)$ & & \\
Never & $37(18)$ & $3(17)$ & \\
Former & $42(21)$ & $7(39)$ & \\
Current & $19(9)$ & $2(11)$ & \\
Missing & $105(52)$ & $6(33)$ & \\
Baseline eGFR & & & \\
Median [IQR] & $44[32-59]$ & $24[19-41]$ & \\
Range & $15-91$ & $15-68$ & \\
\hline
\end{tabular}

$\mathrm{P}$ values were estimated using either a Mann-Whitney $U$-test or Chisquare test, depending on whether continuous or categorical

$e G F R$ estimated glomerular filtration rate (in $\mathrm{mL} / \mathrm{min}$ per $1.72 \mathrm{~m}^{2}$ ), $I Q R$ interquartile range; PCKD, polycystic kidney diseases

intervals between aetiologies, due in part to small sample sizes. Outcomes in the glomerular disease group are variable, due to the heterogeneity of aetiology and response to targeted therapies. The latter in particular may explain the reduced likelihood of progression in this cohort [17].

Baseline eGFR modified the effect of kidney disease aetiology on kidney failure/KRT, as demonstrated by the point estimates in the PCKD group. The PCKD cohort with a baseline eGFR $<45 \mathrm{ml} / \mathrm{min}$ per $1.72 \mathrm{~m}^{2}$ had the highest point estimates for kidney failure/KRT compared with other CKD aetiologies; whereas the PCKD cohort in the higher eGFR group had comparatively lower point estimates compared with diabetic nephropathy, and comparable point estimates to the acquired single kidney cohort. This difference could have a biological basis, due to the multiplicity of mutations associated with PCKD, and may reflect unequal distribution of mutations between the eGFR groups thereby leading to differences in outcomes. For example, mutations at the PKD-1 locus are associated with a higher likelihood of CKD progression compared with PKD-2 [18], and it could be hypothesised that there was a higher proportion of PKD-1 
mutations in the group with the lower eGFR. Genetic information was not available in this cohort, so this hypothesis could not be explored further.

Similar patterns were observed for the outcomes of CKD progression and eGFR change, as for kidney failure/KRT. The diabetic nephropathy and PCKD cohorts in the baseline eGFR $<45 \mathrm{~mL} / \mathrm{min}$ per $1.73 \mathrm{~m}^{2}$ group had higher rates of progression than other cohorts, including acquired single kidney; the remaining groups were comparable. In the eGFR $\geq 45 \mathrm{~mL} / \mathrm{min}$ per $1.73 \mathrm{~m}^{2}$ group, diabetic nephropathy was associated with higher rates of CKD progression and greater reductions in eGFR; whereas the other groups had lower and equivalent estimates.

Collectively, we found that people with diabetic nephropathy were more likely to develop kidney failure/KRT than those with CKD due to an acquired single kidney. Other nonsurgical causes of CKD, such as glomerulonephritis, had rates of kidney failure/KRT and CKD progression that were similar to CKD due to an acquired single kidney. Diabetic nephropathy is the most common cause of CKD in most Western countries [19], and is likely to be disproportionately represented in analyses which combine non-surgical causes of CKD and compare these collectively with surgical causes. This would skew the effect size away from the null hypothesis, and may explain the results of previous studies which reported non-surgical causes of CKD are more likely to progress to kidney failure/KRT than surgical causes [3, 4].

Also notable was the fact that, regardless of underlying aetiology of CKD, risk of adverse outcomes increased substantially with lower eGFR $\left(<45 \mathrm{~mL} / \mathrm{min}\right.$ per $\left.1.72 \mathrm{~m}^{2}\right)$, which is consistent with previous studies [3]. This exemplifies the point that eGFR rather than aetiology of kidney disease should be the driver for risk stratification.

In this cohort, $10 \%$ of the patients with an acquired single kidney developed kidney failure/KRT at a median 19 months after recruitment, and $17 \%$ died at a median 31 months after recruitment. These figures overestimate the expected absolute risk of kidney failure and death among patients with CKD after nephrectomy due to referral bias, and it is reasonable to expect that many patients in this cohort with an acquired single kidney had other risk factors for CKD. Nonetheless, a significant proportion of people in this group experienced adverse outcomes, which is not characteristic of a benign disease process. These results are consistent with a population-based study, also conducted in Queensland, which demonstrated that of 2,739 patients who underwent nephrectomy for kidney cancer between 2009 and 2014, $1.9 \%$ and $1.0 \%$ who underwent either radical or partial nephrectomy went on to develop incident kidney failure within three years of surgery [20].

The strengths of this study lie in its large sample size, and access to information relating to the aetiology of CKD diagnosis, which allowed for a more in-depth comparison of medical and surgical causes of CKD. Limitations include missing data regarding dates of nephrectomy or initial diagnosis of CKD, introducing risk of lead-time and length-time bias. Even with these data comparing surgical causes of CKD with other non-surgical causes, using timeto-event analysis is difficult because surgical causes have a distinct $\mathrm{T}_{0}$, whereas non-surgical causes rarely have a known date of diagnosis, let alone a date of onset/initiating injury. There were limited data available on comorbidities due to variable capture across the included health services, including changes in comorbidities over time, which may have impacted our analysis.

\section{Conclusion}

This study has demonstrated that patients with CKD due to an acquired single kidney have comparable risks of kidney failure and progression to many non-surgical aetiologies of CKD. It is notable that patients with CKD due to diabetic nephropathy who are known to specialist nephrology practices generally had higher likelihood of kidney failure, CKD progression and death, compared with patients who had CKD due to an acquired single kidney. It is likely that the results from previous studies that demonstrate medical causes of CKD as being more aggressive than surgical causes are influenced by the high proportions of patients with diabetic nephropathy in the medical CKD cohorts, which may downplay the risk of surgical CKD. While CKD after nephrectomy is not the most aggressive cause of kidney disease, it is by no means benign, and among patients known to specialist nephrology practices in Queensland, it is associated with a tangible risk of kidney failure and death, that is comparable to other major causes of CKD.

Supplementary Information The online version contains supplementary material available at https://doi.org/10.1007/s11255-022-03114-7.

Acknowledgements With thanks to additional investigators from the NHMRC CKD.CRE and the CKD.QLD Collaborative who were not directly involved in this manuscript: Chris Banney, Ann Bonner, Leanne Brown, Roy Cherian, Luke Connelly, Jeff Coombes, Murthy Divi, Katrina Duff, Zoltan Endre, Robert Fassett, Sridevi Govindarajulu, Stella Green, Nick Gray, Thin Han, Shahadat Hossain, George Kan, Tina Katsanevas, Erica Lennan, Krishan Madhan, Murty Mantha, Valli Manickam, Geoff Mitchell, Chetana Naresh, Kathryn Panaretto, Andrea Pollock, Dwarakanathan Ranganathan, Vikas Srivastava, Ada Stevenson, Thomas Titus, Zaimin Wang, Danielle Wu; and the patients who provided their consent and support for the CKD.QLD Registry.

Funding Open Access funding enabled and organized by CAUL and its Member Institutions. The NHMRC CKD.CRE was funded by a 
National Health and Medical Research Council (Australia) Centres of Research Excellence Grant.

Availability of data and material Data can be obtained on application to the CKD.QLD registry, with appropriate permissions and ethics approvals obtained.

Code availability Data were analysed using Stata 14.2 (StataCorp, College Station, TX) using standard code.

\section{Declarations}

Conflict of interest The authors report no conflicts of interest relevant to this manuscript.

Ethical approval Ethics approval was obtained from the University of Queensland.

Consent to participate All participants provided written informed consent.

Consent for publication Only aggregate data are reported in this manuscript; written informed consent was obtained.

Open Access This article is licensed under a Creative Commons Attribution 4.0 International License, which permits use, sharing, adaptation, distribution and reproduction in any medium or format, as long as you give appropriate credit to the original author(s) and the source, provide a link to the Creative Commons licence, and indicate if changes were made. The images or other third party material in this article are included in the article's Creative Commons licence, unless indicated otherwise in a credit line to the material. If material is not included in the article's Creative Commons licence and your intended use is not permitted by statutory regulation or exceeds the permitted use, you will need to obtain permission directly from the copyright holder. To view a copy of this licence, visit http://creativecommons.org/licenses/by/4.0/.

\section{References}

1. Ljungberg B, Albiges L, Abu-Ghanem Y et al (2019) European association of urology guidelines on renal cell carcinoma: the 2019 update. Eur Urol 75(5):799-810

2. Scosyrev E, Messing EM, Sylvester R, Campbell S, Van Poppel H (2014) Renal function after nephron-sparing surgery versus radical nephrectomy: results from EORTC randomized trial 30904. Eur Urol 65(2):372-377

3. Demirjian S, Lane BR, Derweesh IH, Takagi T, Fergany A, Campbell SC (2014) Chronic kidney disease due to surgical removal of nephrons: relative rates of progression and survival. J Urol 192(4):1057-1062

4. Lane BR, Campbell SC, Demirjian S, Fergany AF (2013) Surgically induced chronic kidney disease may be associated with a lower risk of progression and mortality than medical chronic kidney disease. J Urol 189(5):1649-1655

5. Lane BR, Demirjian S, Derweesh IH, Riedinger CB, Fergany AF, Campbell SC (2014) Is all chronic kidney disease created equal? Curr Opin Urol 24(2):127-134
6. Ellis RJ (2019) Chronic kidney disease after nephrectomy: a clinically-significant entity? Transl Androl Urol 8(Suppl 2):S166-S174

7. O'Hare AM, Batten A, Burrows NR, Pavkov ME, Taylor L, Gupta I et al (2012) Trajectories of kidney function decline in the 2 years before initiation of long-term dialysis. Am J Kidney Dis 59(4):513-522

8. Abeysekera RA, Healy HG, Wang Z, Cameron AL, Hoy WE (2021) Heterogeneity in patterns of progression of chronic kidney disease. Int Med J 51(2):220-228

9. Li L, Astor BC, Lewis J, Hu B, Appel LJ, Lipkowitz MS et al (2012) Longitudinal progression trajectory of GFR among patients with CKD. Am J Kidney Dis 59(4):504-512

10. Tonelli M, Wiebe N, Culleton B, House A, Rabbat C, Fok M et al (2006) Chronic kidney disease and mortality risk: a systematic review. J Am Soc Nephrol 17(7):2034-2047

11. Holzmann MJ, Carlsson AC, Hammar N, Ivert T, Walldius G, Jungner I et al (2016) Chronic kidney disease and 10-year risk of cardiovascular death. Eur J Prev Cardiol 23(11):1187-1194

12. Venuthurupalli SK, Hoy WE, Healy HG, Cameron A, Fassett RG (2017) CKD.QLD: establishment of a chronic kidney disease [CKD] registry in Queensland, Australia. BMC Nephrol 18(1):189

13. Ellis RJ, Del Vecchio SJ, Gallagher KMJ et al (2020) A simple clinical tool for stratifying risk of clinically significant CKD after nephrectomy: development and multinational validation. J Am Soc Nephrol 31(5):1107-1117

14. Ellis RJ, Cho Y, Del Vecchio SJ et al (2019) Outcome measures used to report kidney function in studies investigating surgical management of kidney tumours: a systematic review. Eur Urol Focus 5(6):1074-1084

15. Vallon V, Komers R (2011) Pathophysiology of the diabetic kidney. Compr Physiol 1(3):1175-1232

16. Toth-Manikowski S, Atta MG (2015) Diabetic kidney disease: pathophysiology and therapeutic targets. J Diab Res 2015:697010

17. Wetmore JB, Guo H, Liu J, Collins AJ, Gilbertson DT (2016) The incidence, prevalence, and outcomes of glomerulonephritis derived from a large retrospective analysis. Kidney Int 90(4):853-860

18. Dicks E, Ravani P, Langman D, Davidson WS, Pei Y, Parfrey PS (2006) Incident renal events and risk factors in autosomal dominant polycystic kidney disease: a population and familybased cohort followed for 22 years. Clin J Am Soc Nephrol 1(4):710-717

19. ANZDATA Registry (2017) 39th Report, chapter 1: incidence of end stage kidney disease. Australia and New Zealand Dialysis and Transplant Registry, Adelaide, Australia. Available from: http:// www.anzdata.org.au

20. Ellis RJ, Edey DP, Del Vecchio SJ et al (2018) End-stage kidney disease following surgical management of kidney cancer. Clin J Am Soc Nephrol 13(11):1641-1648

Publisher's Note Springer Nature remains neutral with regard to jurisdictional claims in published maps and institutional affiliations. 